\title{
Delayed Decomposition Approach to Delay-Dependent Stability for Time-Varying Delay Descriptor Systems
}

\author{
Pin-Lin Liu \\ Department of Automation Engineering Institute of Mechatronoptic System, Chienkuo Technology University, Taiwan \\ *Corresponding author: 1p1@cc.ctu.edu.tw
}

Copyright (C) 2013 Horizon Research Publishing All rights reserved.

\begin{abstract}
This paper studies the problem of stability analysis for descriptor systems with time-varying delay. By developing a delayed decomposition approach, information of the delayed plant states can be taken into full consideration, and new delay-dependent sufficient stability criteria are obtained in terms of linear matrix inequalities (LMIs). Then, based on the Lyapunov method, delaydependent stability criteria are devised by taking the relationship between terms in the Leibniz-Newton formula into account. Criteria are derived in terms of LMIs, which can be easily solved by using various convex optimization algorithms. It is proved that the newly proposed criteria may introduce less conservatism than some existing ones. Meanwhile, the computational complexity of the presented stability criteria is reduced greatly since fewer decision variables are involved. Numerical examples are included to show that the proposed method is effective and can provide less conservative results.
\end{abstract}

Keywords Lyapunov-Krasovskii's Functional, Delay Decomposition Approach, Descriptor Systems, Linear Matrix Inequalities (LMIs), Maximum Admissible Upper Bound (MAUB)

\section{Introduction}

Time-delays are often encountered in various dynamic systems, such as manufacturing systems, economic systems, biological systems, networked control systems, and so on. The time-delay is frequently a source of instability and performance deterioration. Therefore, stability analysis and controller synthesis for time-delay system have been one of the most challenging issues [1-31]. The main aim of these studies is to achieve a maximum admissible upper bound (MAUB) such that the system under consideration is globally asymptotically stable for any time delay less than the MAUB. On the other hand, the descriptor system is referred to as a singular system, a generalized state-space system or a semi-state system. It is commonly encountered in many fields such as aircraft attitude control, flexible arm control of robots, large-scale electric network control, chemical engineering systems, lossless transmission lines [2, 4-13, 19-21, 23-28, 30].

Recently, due to the fact that descriptor systems better describe physical systems, many papers have investigated these and some important results have been proposed. To obtain delay-dependent conditions, many efforts have been made in the literature, among which the model transformation technique and bounding technique on cross-product terms are often used [5, 6, 20, 24, 25]. As is well known, these two methods will cause some conservatism. Recently, some improved delay dependent stability criteria have been obtained without using model transformation and bounding techniques for cross terms $[8$, 11, 14-18, 27, 29, 31]. In [8], Jiang et al. proposed free weighting matrices approach to investigate the delay-dependent stability. By employing a Jensen integral inequality technique, $[11,27,29,31]$ further extended the free weighting matrix method. It should be noted that through this methods are effective for the systems with a constant time delay; it can only made a little improvement for the systems with a time-varying delay. Many researchers have realized that many free variables introduced in free weighting matrices method will complicate system analysis, thereby yielding significant increase in computational demand [13]. Hence computational burden involved in solving stability criteria using standard numerical packages becomes minimal.

In [13], an integral inequality was presented for less computational requirement in a alternative stability criterion, and it was intensively used in later literature [14-18]. Later, [14-18] provided integral inequality matrix and delayed decomposition approach, information of the delayed plant states can be taken into full consideration, and new delay-dependent sufficient stability criteria are obtained in terms of linear matrix inequalities (LMIs). Based on the result of [14-18] made some improvements and provided a new integral inequality method to the stability and stabilization analysis of the linear delay-dependent systems, which is proved to be less conservative than the previous 
criteria. Hence computational burden involved in solving stability criteria using standard numerical packages becomes minimal. Furthermore, we not only theoretically prove that our results are less conservative than those in $[6,8,24,27$, $31]$, but also show that our results have higher computational efficiency than those in $[3,6,7,11,21,23-27]$ and some existing results in [14-18] are special cases of our results. Yet when estimating the upper bound of the derivative of Lyapunov functional for descriptor systems with timevarying delay, there is room for investigation.

Motivated by the statement above, this paper proposes improved delay-dependent stability of the descriptor time-varying delay systems studied. An improved delaydependent stability criterion is established in terms of linear matrix inequalities (LMIs), which guarantee the descriptor time-delay system to be regular, impulse free and asymptotically stable. A new delay-dependent stability criterion is derived via a linear matrix inequality formulation that can be easily solved by various convex optimization techniques. The main aim is to derive a MAUB of the time-delay such that the time delay system is asymptotically stable for any delay size less than the MAUB. Accordingly, the obtained MAUB becomes a key performance index to measure the conservatism of a delay-dependent stability condition. We will show that the obtained criterion includes some existing results as its special cases and numerical examples are also given to show the less conservativeness of the presented criterion.

\section{Stability Description and Preliminaries}

Consider the following descriptor system with a time-varying state delay:

$$
\begin{gathered}
E \dot{x}(t)=A x(t)+B x(t-h(t)), \quad t \geq 0, \\
x(t)=\phi(t), \quad t \in[-h, 0],
\end{gathered}
$$

where $x(t) \in R^{n}$ is the state vector, $A$ and $B$ are constant matrices with appropriate dimensions; The matrix $E \in R^{n \times n}$ maybe singular, without loss generality, we suppose rank $E=r \leq n ; \phi(t)$ is a smooth vector-valued initial function. $h(t)$ is a time varying delay in the state, and $h$ is an upper bound on the delay $h(t)$.

We consider two different cases for time varying delays as follows:

Case I: $h(t)$ is a differentiable function, satisfying for all $t \geq 0$ :

$$
0 \leq h(t) \leq h \quad \text { and } \quad \dot{h}(t) \leq h_{d} .
$$

Case II: $h(t)$ is not differentiable or the upper bound of the derivative of $h(t)$ is unknown, and $h(t)$ satisfies

$$
0 \leq h(t) \leq h,
$$

where $h$ and $h_{d}$ are some positive constants.

Here, definitions and fundamental lemmas are reviewed.

Definition 1 (Dai [2]). The pair $(E, A)$ is said to be regular if $\operatorname{det}(s E-A)$ is not identically zero.

Definition 2 (Dai [2]). The pair $(E, A)$ is said to be impulse free if $\operatorname{deg}(\operatorname{det}(s E-A))=\operatorname{rank} E$.

Definition 3. For a given scalar $\bar{h}>0$, the singular delay system (1) is said to be regular and impulse free for any constant time delay $h$ satisfying $0 \leq h \leq \bar{h}$, if the pairs $(E, A)$ and $(E, A+B)$ are regular and impulse free.

Remark 1. The regularity and the absence of impulses of the pair $(E, A)$ ensures the system (1) with time delay $h \neq 0$ to be regular and impulse free, while the fact that the pair $(E, A+B)$ is regular and impulse free ensures the system (1) with time delay $h=0$ to be regular and impulse free.

Lemma 1 (Lewis [9]). The descriptor system $E \dot{x}(t)=A x(t)$ is regular, impulse free, and stable, if and only if there exists a matrix $P$ such that

$$
\begin{gathered}
P^{T} E=E^{T} P \geq 0, \\
A^{T} P+P A<0 .
\end{gathered}
$$

Lemma 2 (Liu [13-18]). For any positive semi-definite matrices

$$
X=\left[\begin{array}{ccc}
X_{11} & X_{12} & X_{13} \\
X_{12}^{T} & X_{22} & X_{23} \\
X_{13}^{T} & X_{23}^{T} & X_{33}
\end{array}\right] \geq 0,
$$

the following integral inequality holds:

$$
\begin{aligned}
& -\int_{t h(t)}^{t} \dot{x}^{T}(s) X_{33} \dot{x}(s) d s \\
& \leq \int_{t-h(t)}^{t}\left[x^{T}(t) \quad x^{T}(t-h(t)) \quad \dot{x}^{T}(s)\right] \\
& {\left[\begin{array}{ccc}
X_{11} & X_{12} & X_{13} \\
X_{12}^{T} & X_{22} & X_{23} \\
X_{13}^{T} & X_{23}^{T} & 0
\end{array}\right]\left[\begin{array}{c}
x(t) \\
x(t-h(t)) \\
\dot{x}(s)
\end{array}\right] d s .}
\end{aligned}
$$

Lemma 3 (Boyd et al. [1]). The following matrix inequality:

$$
\left[\begin{array}{ll}
Q(x) & S(x) \\
S^{T}(x) & R(x)
\end{array}\right]<0
$$

where $Q(x)=Q^{T}(x), R(x)=R^{T}(x)$ and $S(x)$ depend on affine on $x$, is equivalent to

$$
\begin{aligned}
& R(x)<0, \\
& Q(x)<0,
\end{aligned}
$$


and

$$
V\left(x_{t}\right)=V_{1}\left(x_{t}\right)+V_{2}\left(x_{t}\right)+V_{3}\left(x_{t}\right),
$$

$$
Q(x)-S(x) R^{-1}(x) S^{T}(x)<0 .
$$

(6d)

\section{Preliminary Results}

For the system (1)-(2), we will give a stability condition by using a delay decomposition approach as follows:

Theorem 1. In Case I, if $0 \leq h(t) \leq \alpha h$, for given scalars $h(h>0), \alpha(0<\alpha<1)$ and $h_{d}$, the system described by (1) with (2) is asymptotically stable if there exist matrices $P=P^{T}>0, \quad Q_{i}=Q_{i}^{T}>0, \quad R_{i}=R_{i}^{T}>0,(i=1,2,3)$, matrix $S$ of appropriate dimensions and positive semi-definite matrices: $X=\left[\begin{array}{ccc}X_{11} & X_{12} & X_{13} \\ X_{12}^{T} & X_{22} & X_{23} \\ X_{13}^{T} & X_{23}^{T} & X_{33}\end{array}\right] \geq 0$, $Y=\left[\begin{array}{lll}Y_{11} & Y_{12} & Y_{13} \\ Y_{12}^{T} & Y_{22} & Y_{23} \\ Y_{13}^{T} & Y_{23}^{T} & Y_{33}\end{array}\right] \geq 0, Z=\left[\begin{array}{ccc}Z_{11} & Z_{12} & Z_{13} \\ Z_{12}^{T} & Z_{22} & Z_{23} \\ Z_{13}^{T} & Z_{23}^{T} & Z_{33}\end{array}\right] \geq 0$ such that

$$
\Omega=\left[\begin{array}{ccccc}
\Omega_{11} & \Omega_{12} & 0 & 0 & \Omega_{15} \\
\Omega_{12}^{T} & \Omega_{22} & \Omega_{23} & 0 & \Omega_{25} \\
0 & \Omega_{23}^{T} & \Omega_{33} & \Omega_{34} & 0 \\
0 & 0 & \Omega_{34}^{T} & \Omega_{44} & 0 \\
\Omega_{15}^{T} & \Omega_{25}^{T} & 0 & 0 & \Omega_{55}
\end{array}\right]<0,
$$

and

$$
\begin{aligned}
& E^{T} R_{1} E-X_{33} \geq 0, \\
& E^{T} R_{2} E-Y_{33} \geq 0, \\
& E^{T}\left[R_{1}+\left(1-h_{d}\right) R_{3}\right] E-Z_{33} \geq 0
\end{aligned}
$$

where $U \in R^{n \times(n-r)}$ is any matrix satisfying $E^{T} U=0$ and

$$
\begin{aligned}
& \Omega_{11}=A^{T} P+P A+Q_{1}+Q_{3}+A^{T} U S^{T}+S U^{T} A+\alpha h Z_{11}+Z_{13}+Z_{13}^{T}, \\
& \Omega_{12}=P B+S U^{T} B+\alpha h Z_{12}-Z_{13}+Z_{23}^{T}, \\
& \Omega_{15}=A^{T}\left[\alpha h R_{1}+(1-\alpha) h R_{2}+\alpha h R_{3}\right], \\
& \Omega_{22}=-\left(1-h_{d}\right) Q_{3}+\alpha h X_{11}+X_{13}+X_{13}^{T}+\alpha h Z_{22}-Z_{23}-Z_{23}^{T}, \\
& \Omega_{23}=\alpha h X_{12}-X_{13}+X_{23}^{T}, \Omega_{25}=B^{T}\left[\alpha h R_{1}+(1-\alpha) h_{2}+\alpha h R_{3}\right], \\
& \Omega_{33}=Q_{2}-Q_{1}+\alpha h X_{22}-X_{23}-X_{23}^{T}+(1-\alpha) h Y_{11}+Y_{13}+Y_{13}^{T}, \\
& \Omega_{34}=(1-\alpha) h Y_{12}-Y_{13}+Y_{23}^{T}, \Omega_{44}=-Q_{2}+(1-\alpha) h Y_{22}-Y_{23}-Y_{23}^{T}, \\
& \Omega_{55}=-\left[\alpha h R_{1}+(1-\alpha) h R_{2}+\alpha h R_{3}\right] .
\end{aligned}
$$

Proof. In Case I, a Lyapunov functional can be constructed as follows: where

$$
\begin{aligned}
V_{1}\left(x_{t}\right)= & x^{T}(t) P E x(t), \\
V_{2}\left(x_{t}\right)= & \int_{t-\alpha h}^{t} x^{T}(s) Q_{1} x(s) d s+\int_{t-h}^{t-\alpha h} x^{T}(s) Q_{2} x(s) d s \\
& +\int_{t-h(t)}^{t} x^{T}(s) Q_{3} x(s) d s, \\
V_{3}\left(x_{t}\right)= & \int_{-\alpha h}^{0} \int_{t+\theta}^{t} \dot{x}^{T}(s) E^{T} R_{1} E \dot{x}(s) d s d \theta \\
& +\int_{-h}^{-\alpha h} \int_{t+\theta}^{t} \dot{x}^{T}(s) E^{T} R_{2} E \dot{x}(s) d s d \theta \\
& +\int_{-h(t)}^{0} \int_{t+\theta}^{t} \dot{x}^{T}(s) E^{T} R_{3} E \dot{x}(s) d s d \theta .
\end{aligned}
$$

Taking the time derivative of $V\left(x_{t}\right)$ for $t \in[0, \infty)$ along the trajectory of (1), it yields that:

$$
\dot{V}\left(x_{t}\right)=\dot{V}_{1}\left(x_{t}\right)+\dot{V}_{2}\left(x_{t}\right)+\dot{V}_{3}\left(x_{t}\right),
$$

First the derivative of $V_{1}(t)$ is

$$
\begin{aligned}
\dot{V}_{1}\left(x_{t}\right)= & \dot{x}^{T}(t) E^{T} P x(t)+x^{T}(t) P E \dot{x}(t) \\
= & x^{T}(t)\left(A^{T} P+P A\right) x(t)+x^{T}(t) P B x(t-h(t)) \\
& +x^{T}(t-h(t)) B^{T} P x(t),
\end{aligned}
$$

Second, we get the bound of $V_{2}(t)$ as

$$
\begin{aligned}
\dot{V}_{2}\left(x_{t}\right)= & x^{T}(t)\left(Q_{1}+Q_{3}\right) x(t)-x^{T}(t-h(t))(1-\dot{h}(t)) Q_{3} x(t-h(t)) \\
& +x^{T}(t-\alpha h)\left(Q_{2}-Q_{1}\right) x(t-\alpha h)-x^{T}(t-h) Q_{2} x(t-h) \\
& \leq x^{T}(t)\left(Q_{1}+Q_{3}\right) x(t)-x^{T}(t-h(t))\left(1-h_{d}\right) Q_{3} x(t-h(t)) \\
& +x^{T}(t-\alpha h)\left(Q_{2}-Q_{1}\right) x(t-\alpha h)-x^{T}(t-h) Q_{2} x(t-h),
\end{aligned}
$$

Finally, the bound of $V_{3}(t)$ is as follows:

$$
\begin{aligned}
& \dot{V}_{3}\left(x_{t}\right)=\dot{x}^{T}(t) E^{T}\left[\alpha h R_{1}+(1-\alpha) h R_{2}+h(t) R_{3}\right] E \dot{x}(t) \\
& -\int_{t-\alpha h}^{t} \dot{x}^{T}(s) E^{T} R_{1} E \dot{x}(s) d s-\int_{t-h}^{t-\alpha h} \dot{x}^{T}(s) E^{T} R_{2} E \dot{x}(s) d s \\
& -(1-\dot{h}(t)) \int_{t-h(t)}^{t} \dot{x}^{T}(s) E^{T} R_{3} E \dot{x}(s) d s \\
& \leq \dot{x}^{T}(t) E^{T}\left[\alpha h R_{1}+(1-\alpha) h R_{2}+\alpha h R_{3}\right] E \dot{x}(t) \\
& -\int_{t-\alpha h}^{t} \dot{x}^{T}(s) E^{T} R_{1} E \dot{x}(s) d s-\int_{t-h}^{t-\alpha h} \dot{x}^{T}(s) E^{T} R_{2} E \dot{x}(s) d s \\
& -\left(1-h_{d}\right) \int_{t-h(t)}^{t} \dot{x}^{T}(s) E^{T} R_{3} E \dot{x}(s) d s .
\end{aligned}
$$

Now, we estimate the upper bound of the last three terms in inequality (12) as follows: 
$-\int_{t-\alpha h}^{t} \dot{x}^{T}(s) E^{T} R_{1} E \dot{x}(s) d s-\int_{t-h}^{t-\alpha h} \dot{x}^{T}(s) E^{T} R_{2} E \dot{x}(s) d s$

$-\left(1-h_{d}\right) \int_{t-h(t)}^{t} \dot{x}^{T}(s) E^{T} R_{3} E \dot{x}(s) d s$

$=-\int_{t-\alpha h}^{t-h(t)} \dot{x}^{T}(s) E^{T} R_{1} E \dot{x}(s) d s-\int_{t-h}^{t-\alpha h} \dot{x}^{T}(s) E^{T} R_{2} E \dot{x}(s) d s$

$-\int_{t-h(t)}^{t} \dot{x}^{T}(s) E^{T}\left[R_{1}+\left(1-h_{d}\right) R_{3}\right] E^{T} \dot{x}(s) d s$

$=-\int_{t-\alpha h}^{t-h(t)} \dot{x}^{T}(s)\left(E^{T} R_{1} E-X_{33}\right) \dot{x}(s) d s$

$-\int_{t-h}^{t-\alpha h} \dot{x}^{T}(s)\left(E^{T} R_{2} E-Y_{33}\right) \dot{x}(s) d s$

$-\int_{t-h(t)}^{t} \dot{x}^{T}(s)\left\{E^{T}\left[R_{1}+\left(1-h_{d}\right) R_{3}\right] E-Z_{33}\right\} \dot{x}(s) d s$

$-\int_{t-\alpha h}^{t-h(t)} \dot{x}^{T}(s) X_{33} \dot{x}(s) d s-\int_{t-h}^{t-\alpha h} \dot{x}^{T}(s) Y_{33} \dot{x}(s) d s$

$-\int_{t-h(t)}^{t} \dot{x}^{T}(s) Z_{33} \dot{x}(s) d s$

From Lemma 2, noticing that $E^{T} R_{1} E-X_{33} \geq 0$, $E^{T} R_{2} E-Y_{33} \geq 0, \quad$ and $\quad E^{T}\left[R_{1}+\left(1-h_{d}\right) R_{3}\right] E-Z_{33} \geq 0, \quad$ it yields that:

$$
\begin{aligned}
& -\int_{t-\alpha h}^{t-h(t)} \dot{x}^{T}(s) X_{33} \dot{x}(s) d s \\
& \leq \int_{t-\alpha h}^{t-h(t)}\left[x^{T}(t-h(t)) \quad x^{T}(t-\alpha h) \quad \dot{x}^{T}(s)\right] \times \\
& {\left[\begin{array}{ccc}
X_{11} & X_{12} & X_{13} \\
X_{12}^{T} & X_{22} & X_{23} \\
X_{13}^{T} & X_{23}^{T} & 0
\end{array}\right]\left[\begin{array}{c}
x(t-h(t)) \\
x(t-\alpha h) \\
\dot{x}(s)
\end{array}\right] d s}
\end{aligned}
$$

$\leq x^{T}(t-h(t))(\alpha h-h(t)) X_{11} x(t-h(t))$

$+x^{T}(t-h(t))(\alpha h-h(t)) X_{12} x(t-\alpha h)$

$+x^{T}(t-h(t)) X_{13} t_{t-\alpha h}^{t-h(t)} \dot{x}(s) d s$

$+x^{T}(t-\alpha h)(\alpha h-h(t)) X_{12}^{T} x(t-h(t))$

$+x^{T}(t-\alpha h)(\alpha h-h(t)) X_{22} x(t-\alpha h)$

$+x^{T}(t-\alpha h) X_{23}{ }_{t-\alpha h}^{t-h(t)} \dot{x}(s) d s$

$+\int_{t-\alpha h}^{t-h(t)} \dot{x}^{T}(s) d s X_{13}^{T} x(t-h(t))$

$+\int_{t-\alpha h}^{t-h(t)} \dot{x}^{T}(s) d s X_{23}^{T} x(t-\alpha h)$

$\leq x^{T}(t-h(t)) \alpha h_{X_{1}} x(t-h(t))+x^{T}(t-h(t)) \alpha h_{X_{12}} x(t-\alpha h)$

$+x^{T}(t-h(t)) X_{13} \int_{t-\alpha h}^{t-h(t)} \dot{x}(s) d s+x^{T}(t-\alpha h) \alpha h X_{12}^{T} x(t-h(t))$

$+x^{T}(t-\alpha h) \alpha h X_{22} x(t-\alpha h)+x^{T}(t-\alpha h) X_{23} \int_{t-\alpha h}^{t-h(t)} \dot{x}(s) d s$

$+\int_{t-\alpha h}^{t-h(t)} \dot{x}^{T}(s) d s X_{13}^{T} x(t-h(t))+\int_{t-\alpha h}^{t-h(t)} \dot{x}^{T}(s) d s X_{23}^{T} x(t-\alpha h)$

$=x^{T}(t-h(t))\left[\alpha h X_{11}+X_{13}^{T}+X_{13}\right] x(t-h(t))$

$+x^{T}(t-h(t))\left[\alpha h X_{12}-X_{13}+X_{23}^{T}\right] x(t-\alpha h)$

$+x^{T}(t-\alpha h)\left[\alpha h X_{12}^{T}-X_{13}^{T}+X_{23}\right] x(t-h(t))$

$+x^{T}(t-\alpha h)\left[\alpha h X_{22}-X_{23}-X_{23}^{T}\right] x(t-\alpha h)$.

Similarly, we obtain

$$
\begin{aligned}
& -\int_{t-h}^{t-\alpha h} \dot{x}^{T}(s) Y_{33} \dot{x}(s) d s \\
& \leq x^{T}(t-\alpha h)\left[(1-\alpha) h Y_{11}+Y_{13}^{T}+Y_{13}\right] x(t-\alpha h) \\
& +x^{T}(t-\alpha h)\left[(1-\alpha) h Y_{12}-Y_{13}+Y_{23}^{T}\right] x(t-h) \\
& +x^{T}(t-h)\left[(1-\alpha) h Y_{12}^{T}-Y_{13}^{T}+Y_{23}\right] x(t-\alpha h) \\
& +x^{T}(t-h)\left[(1-\alpha) h Y_{22}-Y_{23}-Y_{23}^{T}\right] x(t-h),
\end{aligned}
$$

$$
\begin{aligned}
& -\int_{t-h(t)}^{t} \dot{x}^{T}(s) Z_{33} \dot{x}(s) d s \\
& \leq x^{T}(t)\left[h(t) Z_{11}+Z_{13}^{T}+Z_{13}\right] x(t) \\
& +x^{T}(t)\left[h(t) Z_{12}-Z_{13}+Z_{23}^{T}\right] x(t-h(t)) \\
& +x^{T}(t-h(t))\left[h(t) h Z_{12}^{T}-Z_{13}^{T}+Z_{23}\right] x(t) \\
& +x^{T}(t-h(t))\left[h(t) Z_{22}-Z_{23}-Z_{23}^{T}\right] x(t-h(t)) \\
& \leq x^{T}(t)\left[\alpha h Z_{11}+Z_{13}^{T}+Z_{13}\right] x(t) \\
& +x^{T}(t)\left[\alpha h Z_{12}-Z_{13}+Z_{23}^{T}\right] x(t-h(t)) \\
& +x^{T}(t-h(t))\left[\alpha h Z_{12}^{T}-Z_{13}^{T}+Z_{23}\right] x(t) \\
& +x^{T}(t-h(t))\left[\alpha h Z_{22}-Z_{23}-Z_{23}^{T}\right] x(t-h(t)) .
\end{aligned}
$$

The operator for the term

$\dot{x}^{T}(t) E^{T}\left[\alpha h_{R_{1}}+(1-\alpha) h_{R_{2}}+\alpha h R_{3}\right] E \dot{x}(t)$ is as follows:

$$
\begin{aligned}
& \quad \dot{x}^{T}(t) E^{T}\left[\alpha h_{1}+(1-\alpha) h_{R_{2}}+\alpha h_{R_{3}}\right] E \dot{x}(t) \\
& =[A x(t)+B x(t-h(t))]^{T}\left[\alpha h R_{1}+(1-\alpha) h R_{2}+\alpha h R_{3}\right] \times \\
& \quad[A x(t)+B x(t-h(t))] \\
& =x^{T}(t) A^{T}\left[\alpha h R_{1}+(1-\alpha) h R_{2}+\alpha h R_{3}\right] A x(t) \\
& +x^{T}(t) A^{T}\left[\alpha h R_{1}+(1-\alpha) h R_{2}+\alpha h R_{3}\right] B x(t-h(t)) \\
& +x^{T}(t-h(t)) B^{T}\left[\alpha h R_{1}+(1-\alpha) h_{R_{2}}+\alpha h R_{3}\right] A x(t) \\
& +x^{T}(t-h(t)) B^{T}\left[\alpha h R_{1}+(1-\alpha) h_{R_{2}}+\alpha h R_{3}\right] B x(t-h(t)) . \quad(17)
\end{aligned}
$$

Furthermore, noting $E^{T} U=0$, we can deduce

$$
0=2 \dot{x}^{T}(t) E^{T} U S^{T} x(t) .
$$

Combining (9)-(18), it yields:

$$
\begin{aligned}
& \dot{V}\left(x_{t}\right) \leq \xi^{T}(t) \Xi \xi(t)-\int_{t-\alpha h}^{t-h(t)} \dot{x}^{T}(s)\left(E^{T} R_{1} E-X_{33}\right) \dot{x}(s) d s \\
& -\int_{t-h}^{t-\alpha h} \dot{x}^{T}(s)\left(E^{T} R_{2} E-Y_{33}\right) \dot{x}(s) d s \\
& -\int_{t-h(t)}^{t} \dot{x}^{T}(s)\left\{E^{T}\left[R_{1}+\left(1-h_{d}\right) R_{3}\right] E-Z_{33}\right\} \dot{x}(s) d s
\end{aligned}
$$

where $\quad \xi^{T}(t)=\left[\begin{array}{llll}x^{T}(t) & x^{T}(t-h(t)) & x^{T}(t-\alpha h) & x^{T}(t-h)\end{array}\right]$

$$
\begin{aligned}
& \text { and } \Xi=\left[\begin{array}{cccc}
\Xi_{11} & \Xi_{12} & 0 & 0 \\
\Xi_{12}^{T} & \Xi_{22} & \Xi_{23} & 0 \\
0 & \Xi_{23}^{T} & \Xi_{33} & \Xi_{34} \\
0 & 0 & \Xi_{34}^{T} & \Xi_{44}
\end{array}\right] \text {, with } \\
& \Xi_{11}=A^{T} P+P A+Q_{1}+Q_{3}+A^{T} U S^{T}+S U^{T} A+\alpha h Z_{11}+Z_{13}+Z_{13}^{T} \text {, } \\
& +A^{T}\left[\alpha h_{R_{1}}+(1-\alpha) h_{R_{2}}+\alpha h_{R_{3}}\right] A, \\
& \Xi_{12}=P B+S U^{T} B+\alpha h Z_{12}-Z_{13}+Z_{23}^{T} \\
& +A^{T}\left[\alpha h_{R_{1}}+(1-\alpha) h_{R_{2}}+\alpha h_{R_{3}}\right] B, \\
& \Xi_{22}=-\left(1-h_{d}\right) Q_{3}+h \alpha X_{11}+X_{13}+X_{13}^{T}+\alpha h Z_{22}-Z_{23}-Z_{23}^{T} \text {, } \\
& +B^{T}\left[\alpha h_{R_{1}}+(1-\alpha) h_{R_{2}}+\alpha h_{R_{3}}\right] B, \\
& \Xi_{23}=\alpha h_{X_{12}}-X_{13}+X_{23}^{T} \text {, } \\
& \Xi_{33}=Q_{2}-Q_{1}+\alpha h X_{22}-X_{23}-X_{23}^{T}+(1-\alpha) h Y_{11}+Y_{13}+Y_{13}^{T} \text {, } \\
& \Xi_{34}=(1-\alpha) h Y_{12}-Y_{13}+Y_{23}^{T} \text {, } \\
& \Xi_{44}=-Q_{2}+(1-\alpha) h Y_{22}-Y_{23}-Y_{23}^{T} \text {. }
\end{aligned}
$$

From Equation (1) and the Schur complement of Lemma 3, it is easy to see that $\dot{V}\left(x_{t}\right)<0$ holds if $E^{T} R_{1} E-X_{33} \geq 0, E^{T} R_{2} E-Y_{33} \geq 0$, 
$E^{T}\left[R_{1}+\left(1-h_{d}\right) R_{3}\right] E-Z_{33} \geq 0$, and $0 \leq h(t) \leq \alpha h$.

Theorem 2. In Case I, if $\alpha h \leq h(t) \leq h$, for given scalars $h(h>0), \alpha(0<\alpha<1)$ and $h_{d}$, the system described by (1) with (2) is asymptotically stable if there exist matrices $P=P^{T}>0, Q_{i}=Q_{i}^{T}>0, R_{i}=R_{i}^{T}>0,(i=1,2,3)$, matrix $S$ of appropriate dimensions and $X=\left[\begin{array}{ccc}X_{11} & X_{12} & X_{13} \\ X_{12}^{T} & X_{22} & X_{23} \\ X_{13}^{T} & X_{23}^{T} & X_{33}\end{array}\right] \geq 0$,

$$
Y=\left[\begin{array}{lll}
Y_{11} & Y_{12} & Y_{13} \\
Y_{12}^{T} & Y_{22} & Y_{23} \\
Y_{13}^{T} & Y_{23}^{T} & Y_{33}
\end{array}\right] \geq 0, Z=\left[\begin{array}{ccc}
Z_{11} & Z_{12} & Z_{13} \\
Z_{12}^{T} & Z_{22} & Z_{23} \\
Z_{13}^{T} & Z_{23}^{T} & Z_{33}
\end{array}\right] \geq 0 \quad \text { such }
$$

that

$$
\bar{\Omega}=\left[\begin{array}{ccccc}
\bar{\Omega}_{11} & \bar{\Omega}_{12} & \bar{\Omega}_{13} & 0 & \bar{\Omega}_{15} \\
\bar{\Omega}_{12}^{T} & \bar{\Omega}_{22} & \bar{\Omega}_{23} & \bar{\Omega}_{24} & \bar{\Omega}_{25} \\
\bar{\Omega}_{13}^{T} & \bar{\Omega}_{23}^{T} & \bar{\Omega}_{33} & 0 & 0 \\
0 & \bar{\Omega}_{24}^{T} & 0 & \bar{\Omega}_{44} & 0 \\
\bar{\Omega}_{15}^{T} & \bar{\Omega}_{25}^{T} & 0 & 0 & \bar{\Omega}_{55}
\end{array}\right]<0,
$$

and

$$
\begin{aligned}
& E^{T}\left[R_{1}+\left(1-h_{d}\right) R_{3}\right] E-X_{33} \geq 0, \\
& E^{T}\left[R_{2}+\left(1-h_{d}\right) R_{3}\right] E-Y_{33} \geq 0, \\
& E^{T} R_{2} E-Z_{33} \geq 0
\end{aligned}
$$

where $U \in R^{n \times(n-r)}$ is any matrix satisfying $E^{T} U=0$ and

$$
\begin{aligned}
\bar{\Omega}_{11}= & A^{T} P+P A+Q_{1}+Q_{3}+A^{T} U S^{T}+S U^{T} A \\
& +\alpha h X_{11}+X_{13}+X_{13}^{T}, \\
\bar{\Omega}_{12} & =P B+S U^{T} B, \\
\bar{\Omega}_{13}= & \alpha h X_{12}-X_{13}+X_{23}^{T}, \\
& \bar{\Omega}_{15}=A^{T}\left[\alpha h R_{1}+(1-\alpha) h R_{2}+h R_{3}\right], \\
\bar{\Omega}_{22}= & -\left(1-h_{d}\right) Q_{3}+(1-\alpha) h Y_{22}-Y_{23}-Y_{23}^{T} \\
& +(1-\alpha) h Z_{11}+Z_{13}+Z_{13}^{T},
\end{aligned}
$$

$$
\begin{aligned}
& \left.\bar{\Omega}_{23}=1-\alpha\right) h Y_{12}^{T}-Y_{13}^{T}+Y_{23}, \\
& \bar{\Omega}_{24}=(1-\alpha) h Z_{12}-Z_{13}+Z_{23}^{T}, \\
& \bar{\Omega}_{25}=B^{T}\left[\alpha h R_{1}+(1-\alpha) h R_{2}+h R_{3}\right], \\
& \bar{\Omega}_{33}=Q_{2}-Q_{1}+\alpha h X_{22}-X_{23}-X_{23}^{T}+(1-\alpha) h Y_{11}+Y_{13}+Y_{13}^{T}, \\
& \bar{\Omega}_{44}=-Q_{2}+(1-\alpha) h Z_{22}-Z_{23}-Z_{23}^{T}, \\
& \bar{\Omega}_{55}=-\left[\alpha h R_{1}+(1-\alpha) h R_{2}+h R_{3}\right], .
\end{aligned}
$$

Proof. If $\alpha h \leq h(t) \leq h$, it gets: $-\int_{t-\alpha h}^{t} \dot{x}^{T}(s) E^{T} R_{1} E \dot{x}(s) d s-\int_{t-h}^{t-\alpha h} \dot{x}^{T}(s) E^{T} R_{2} E \dot{x}(s) d s$ $-\left(1-h_{d}\right) \int_{t-h(t)}^{t} \dot{x}^{T}(s) E^{T} R_{3} E \dot{x}(s) d s$ $=-\int_{t-\alpha h}^{t} \dot{x}^{T}(s) E^{T}\left[R_{1}+\left(1-h_{d}\right) R_{3}\right] E \dot{x}(s) d s$ $-\int_{t-h(t)}^{t-\alpha h} \dot{x}^{T}(s) E^{T}\left[R_{2}+\left(1-h_{d}\right) R_{3}\right] E \dot{x}(s) d s$ $-\int_{t-h}^{t-h(t)} \dot{x}^{T}(s) E^{T} R_{2} E \dot{x}(s) d s$

$=-\int_{t-\alpha h}^{t} \dot{x}^{T}(s)\left\{E^{T}\left[R_{1}+\left(1-h_{d}\right) R_{3}\right] E-X_{33}\right\} \dot{x}(s) d s$ $-\int_{t-h(t)}^{t-\alpha h} \dot{x}^{T}(s)\left\{E^{T}\left[R_{2}+\left(1-h_{d}\right) R_{3}\right] E-Y_{33}\right\} \dot{x}(s) d s$ $-\int_{t-h}^{t-h(t)} \dot{x}^{T}(s)\left(E^{T} R_{2} E-Z_{33}\right) \dot{x}(s) d s-\int_{t-\alpha h}^{t} \dot{x}^{T}(s) X_{33} \dot{x}(s) d s$ $-\int_{t-h(t)}^{t-\alpha h} \dot{x}^{T}(s) Y_{33} \dot{x}(s) d s-\int_{t-h}^{t-h(t)} \dot{x}^{T}(s) Z_{33} \dot{x}(s) d s$

From Lemma 2, noticing that $E^{T}\left[R_{1}+\left(1-h_{d}\right) R_{3}\right] E-X_{33} \geq 0$, $E^{T}\left[R_{2}+\left(1-h_{d}\right) R_{3}\right] E-Y_{33} \geq 0$, and $E^{T} R_{2} E-Z_{33} \geq 0$, it yields that

$$
\begin{aligned}
& -\int_{t-\alpha h}^{t} \dot{x}^{T}(s) X_{33} \dot{x}(s) d s \\
& \leq x^{T}(t)\left[\alpha h X_{11}+X_{13}^{T}+X_{13}\right] x(t) \\
& +x^{T}(t)\left[\alpha h X_{12}-X_{13}+X_{23}^{T}\right] x(t-\alpha h) \\
& +x^{T}(t-\alpha h)\left[\alpha h X_{12}^{T}-X_{13}^{T}+X_{23}\right] x(t) \\
& +x^{T}(t-\alpha h)\left[\alpha h X_{22}-X_{23}-X_{23}^{T}\right] x(t-\alpha h) \\
& -\int_{t-h(t)}^{t-\alpha h} \dot{x}^{T}(s) Y_{33} \dot{x}(s) d s \\
& \leq x^{T}(t-\alpha h)\left[(1-\alpha) h Y_{11}+Y_{13}^{T}+Y_{13}\right] x(t-\alpha h) \\
& +x^{T}(t-\alpha h)\left[(1-\alpha) h Y_{12}-Y_{13}+Y_{23}^{T}\right] x(t-h(t)) \\
& +x^{T}(t-h(t))\left[(1-\alpha) h Y_{12}^{T}-Y_{13}^{T}+Y_{23}\right] x(t-\alpha h) \\
& +x^{T}(t-h(t))\left[(1-\alpha) h Y_{22}-Y_{23}-Y_{23}^{T}\right] x(t-h(t))
\end{aligned}
$$

and

$$
\begin{aligned}
& -\int_{t-h}^{t-h(t)} \dot{x}^{T}(s) Z_{33} \dot{x}(s) d s \\
& \leq x^{T}(t-h(t))\left[(1-\alpha) h Z_{11}+Z_{13}^{T}+Z_{13}\right] x(t-h(t)) \\
& +x^{T}(t-h(t))\left[(1-\alpha) h Z_{12}-Z_{13}+Z_{23}^{T}\right] x(t-h) \\
& +x^{T}(t-h)\left[(1-\alpha) h Z_{12}^{T}-Z_{13}^{T}+Z_{23}\right] x(t-h(t)) \\
& +x^{T}(t-h)\left[(1-\alpha) h Z_{22}-Z_{23}-Z_{23}^{T}\right] x(t-h)
\end{aligned}
$$

Combining (9)-(18) and (22)-(24) yields

$$
\begin{aligned}
& \dot{V}\left(x_{t}\right) \leq \xi^{T}(t) \bar{\Xi} \xi(t) \\
& \left.\left.-\int_{t-\alpha h}^{t} \dot{x}^{T}(s)\left\{E^{T}\left[R_{1}+\left(1-h_{d}\right) R_{3}\right)\right] E-X_{33}\right\} \dot{x}(s) d s\right) \\
& -\int_{t-\alpha h(t)}^{t-\dot{x}^{T}}(s)\left\{E^{T}\left[R_{2}+\left(1-h_{d}\right) R_{3}\right] E-Y_{33}\right\} \dot{x}(s) d s \\
& -\int_{t-h}^{t-h(t)} \dot{x}^{T}(s)\left(E^{T} R_{2} E-Z_{33}\right) \dot{x}(s) d s
\end{aligned}
$$




$$
\bar{\Xi}_{11}=A^{T} P+P A+Q_{1}+Q_{3}+A^{T} U S^{T}+S U^{T} A
$$

$+\alpha h X_{11}+X_{13}+X_{13}^{T},+A^{T}\left[\alpha h R_{1}+(1-\alpha) h R_{2}+h R_{3}\right] A$,

$\bar{\Xi}_{12}=P B+S U^{T} B+A^{T}\left[\alpha h_{R_{1}}+(1-\alpha) h_{R_{2}}+h_{R_{3}}\right] B$,

$\bar{\Xi}_{13}=\alpha h_{12}-X_{13}+X_{23}^{T}$,

$\left.\bar{\Xi}_{22}=-\left(1-h_{d}\right) Q_{3}+1-\alpha\right) h Y_{22}-Y_{23}-Y_{23}^{T}+(1-\alpha) h Z_{11}+Z_{13}+Z_{13}^{T}$

$+B^{T}\left[\alpha h R_{1}+(1-\alpha) h R_{2}+h R_{3}\right] B$,

$\left.\bar{\Xi}_{23}=(1-\alpha) h Y_{12}^{T}-Y_{13}^{T}+Y_{23}, \bar{\Xi}_{24}=1-\alpha\right) h Z_{12}-Z_{13}+Z_{23}^{T}$,

$\bar{\Xi}_{33}=Q_{2}-Q_{1}+\alpha h_{X_{22}}-X_{23}-X_{23}^{T}+(1-\alpha) h Y_{11}^{T}+Y_{13}+Y_{13}^{T}$,

$\bar{\Xi}_{44}=-Q_{2}+(1-\alpha) h Z_{22}-Z_{23}-Z_{23}^{T}$.

From Equation (1) and the Schur complement of Lemma 3, it is easy to see that $\dot{V}\left(x_{t}\right)<0$ holds if $E^{T}\left[R_{1}+\left(1-h_{d}\right) R_{3}\right] E-X_{33} \geq 0, E^{T}\left[R_{2}+\left(1-h_{d}\right) R_{3}\right] E-Y_{33} \geq 0$, $E^{T} R_{2} E-Z_{33} \geq 0$, and $\alpha h \leq h(t) \leq h$.

Theorem 3. In Case II, if $0 \leq h(t) \leq \alpha h$, for given scalars $h(h>0), \alpha(0<\alpha<1)$, the system described by (1) with (3) is asymptotically stable if there exist matrices $P=P^{T}>0$,

$Q_{i}=Q_{i}^{T}>0, R_{i}=R_{i}^{T}>0,(i=1,2)$, matrix $S$ of appropriate dimensions and positive semi-definite matrices

$$
\begin{aligned}
X & =\left[\begin{array}{lll}
X_{11} & X_{12} & X_{13} \\
X_{12}^{T} & X_{22} & X_{23} \\
X_{13}^{T} & X_{23}^{T} & X_{33}
\end{array}\right] \geq 0, \quad Y=\left[\begin{array}{ccc}
Y_{11} & Y_{12} & Y_{13} \\
Y_{12}^{T} & Y_{22} & Y_{23} \\
Y_{13}^{T} & Y_{23}^{T} & Y_{33}
\end{array}\right] \geq 0, \\
Z & =\left[\begin{array}{lll}
Z_{11} & Z_{12} & Z_{13} \\
Z_{12}^{T} & Z_{22} & Z_{23} \\
Z_{13}^{T} & Z_{23}^{T} & Z_{33}
\end{array}\right] \geq 0 \text { such that }
\end{aligned}
$$$$
P^{T} E=E^{T} P \geq 0,
$$$$
\Psi=\left[\begin{array}{ccccc}
\Psi_{11} & \Psi_{12} & 0 & 0 & \Psi_{15} \\
\Psi_{12}^{T} & \Psi_{22} & \Psi_{23} & 0 & \Psi_{25} \\
0 & \Psi_{23}^{T} & \Psi_{33} & \Psi_{34} & 0 \\
0 & 0 & \Psi_{34}^{T} & \Psi_{44} & 0 \\
\Psi_{15}^{T} & \Psi_{25}^{T} & 0 & 0 & \Psi_{55}
\end{array}\right]<0
$$$$
E^{T} R_{1} E-X_{33} \geq 0, E^{T} R_{2} E-Y_{33} \geq 0, E^{T} R_{1} E-Z_{33} \geq 0
$$

where $U \in R^{n \times(n-r)}$ is any matrix satisfying $E^{T} U=0$ and

$$
\begin{aligned}
\Psi_{11} & =A^{T} P+P A+Q_{1}+A^{T} U S^{T}+S U^{T} A+h Z_{11}+Z_{13}+Z_{13}^{T}, \\
\Psi_{12} & =P B+S U^{T} B+h Z_{12}-Z_{13}+Z_{23}^{T}, \\
\Psi_{15} & =A^{T}\left[\alpha h R_{1}+(1-\alpha) h R_{2}\right], \\
\Psi_{22} & =\alpha h X_{11}+X_{13}+X_{13}^{T}+h Z_{22}-Z_{23}-Z_{23}^{T}, \\
\Psi_{23} & =\alpha h X_{12}-X_{13}+X_{23}^{T}, \\
\Psi_{25} & =B^{T}\left[\alpha h R_{1}+(1-\alpha) h R_{2}\right], \\
\Psi_{33} & =Q_{2}-Q_{1}+\alpha h X_{22}-X_{23}-X_{23}^{T}+(1-\alpha) h Y_{11}+Y_{13}+Y_{13}^{T}, \\
\Psi_{34} & =(1-\alpha) h Y_{12}-Y_{13}+Y_{23}^{T}, \\
\Psi_{44} & =-Q_{2}+(1-\alpha) h Y_{22}-Y_{23}-Y_{23}^{T},
\end{aligned}
$$

$$
\Psi_{55}=-\left[\alpha h_{R_{1}}+(1-\alpha) h_{R_{2}}\right] .
$$

Theorem 4. In Case II, if $\alpha h \leq h(t) \leq h$, for given scalars $h(h>0), \alpha(0<\alpha<1)$, the system described by (1) with (3) is asymptotically stable if there exist matrices $P=P^{T}>0$, $Q_{i}=Q_{i}^{T}>0, R_{i}=R_{i}^{T}>0,(i=1,2)$, matrix $S$ of appropriate dimensions and positive semi-definite matrices $X=\left[\begin{array}{ccc}X_{11} & X_{12} & X_{13} \\ X_{12}^{T} & X_{22} & X_{23} \\ X_{13}^{T} & X_{23}^{T} & X_{33}\end{array}\right] \geq 0, \quad Y=\left[\begin{array}{ccc}Y_{11} & Y_{12} & Y_{13} \\ Y_{12}^{T} & Y_{22} & Y_{23} \\ Y_{13}^{T} & Y_{23}^{T} & Y_{33}\end{array}\right] \geq 0$, $Z=\left[\begin{array}{ccc}Z_{11} & Z_{12} & Z_{13} \\ Z_{12}^{T} & Z_{22} & Z_{23} \\ Z_{13}^{T} & Z_{23}^{T} & Z_{33}\end{array}\right] \geq 0$ such that

$$
P^{T} E=E^{T} P \geq 0,
$$

$$
\bar{\Psi}=\left[\begin{array}{ccccc}
\bar{\Psi}_{11} & \bar{\Psi}_{12} & \bar{\Psi}_{13} & 0 & \bar{\Psi}_{15} \\
\bar{\Psi}_{12}^{T} & \bar{\Psi}_{22} & \bar{\Psi}_{23} & \bar{\Psi}_{24} & \bar{\Psi}_{25} \\
\bar{\Psi}_{13}^{T} & \bar{\Psi}_{23}^{T} & \bar{\Psi}_{33} & 0 & 0 \\
0 & \bar{\Psi}_{24}^{T} & 0 & \bar{\Psi}_{44} & 0 \\
\bar{\Psi}_{15}^{T} & \bar{\Psi}_{25}^{T} & 0 & 0 & \bar{\Psi}_{55}
\end{array}\right]<0
$$

and

$$
\begin{aligned}
& E^{T} R_{1} E-X_{33} \geq 0, \\
& E^{T} R_{2} E-Y_{33} \geq 0, \\
& E^{T} R_{2} E-Z_{33} \geq 0,
\end{aligned}
$$

where $U \in R^{n \times(n-r)}$ is any matrix satisfying $E^{T} U=0$ and

$$
\begin{aligned}
& \bar{\Psi}_{11}=A^{T} P+P A+Q_{1}+A^{T} U S^{T}+S U^{T} A+\alpha h X_{11}+X_{13}+X_{13}^{T}, \\
& \bar{\Psi}_{12}=P B+S U^{T} B, \bar{\Psi}_{13}=\alpha h X_{12}-X_{13}+X_{23}^{T}, \\
& \bar{\Psi}_{15}=A^{T}\left[\alpha h R_{1}+(1-\alpha) h R_{2}\right], \\
& \bar{\Psi}_{22}=(1-\alpha) h Y_{22}-Y_{23}-Y_{23}^{T}+(1-\alpha) h Z_{11}+Z_{13}+Z_{13}^{T}, \\
& \bar{\Psi}_{23}=(1-\alpha) h Y_{12}^{T}-Y_{13}^{T}+Y_{23}, \bar{\Psi}_{24}=(1-\alpha) h Z_{12}-Z_{13}+Z_{23}^{T}, \\
& \bar{\Psi}_{25}=B^{T}\left[\alpha h R_{1}+(1-\alpha) h R_{2}\right], \\
& \bar{\Psi}_{33}=Q_{2}-Q_{1}+\alpha h X_{22}-X_{23}-X_{23}^{T}+(1-\alpha) h Y_{11}+Y_{13}+Y_{13}^{T}, \\
& \bar{\Psi}_{44}=-Q_{2}+(1-\alpha) h Z_{22}-Z_{23}-Z_{23}^{T}, \\
& \bar{\Psi}_{55}=-\left[\alpha h R_{1}+(1-\alpha) h R_{2}\right] .
\end{aligned}
$$

In Case II, a Lyapunov functional can be chosen as (8) with $Q_{3}=R_{3}=0$. Similar to the above analysis, one can get that $\dot{V}\left(x_{t}\right)<0$ holds if $\Psi<0(\bar{\Psi}<0)$. Thus, the proof is completed.

Remark 1. In the proof of Theorems 1-4, the interval $[t-h, t]$ is divided into two subintervals $[t-h, t-\alpha h]$ and $[t-\alpha h, t]$, the information of delayed state $x(t-\alpha h)$ can be taken into account. It is clear that the Lyapunov function defined in Theorems 1-4 are more general than the ones in [6, $10,21,24,27]$. 
Remark 2. In the previous works except [4, 5, 6, 19, 24, 25], the time delay term $h(t)$ was usually estimated as $h$ when estimating the upper bound of some cross term, this may lead to increasing conservatism inevitably. In Theorems 1-4, the value of the upper bound of some cross term is estimated more exactly than the previous methods since $h(t)$ is confined to the subintervals $0 \leq h(t) \leq \alpha h \quad(\alpha h \leq h(t) \leq h)$. So, such decomposition method may lead to reduction of conservatism.

Remark 3. In the stability problem, MAUB $\bar{h}$ which ensures that singular system with a time-varying state delay (1) is stabilizable for any $h$ can be determined by solving the following quasi-convex optimization problem when the other bound of time-varying delay $h$ is known.

$$
\left\{\begin{array}{lc}
\text { Maximize } & \bar{h} \\
\text { Subject to } & \text { Theorems 1-4. }
\end{array}\right.
$$

Inequality (28) is a convex optimization problem and can be obtained efficiently using the MATLAB LMI Toolbox.

For seeking an appropriate a satisfying $0<\alpha<1$, such that the upper bound $h$ of delay $h(t)$ subjecting to (7) is maximal, we develop an algorithm:

Algorithm 1. (Maximizing $h>0$ )

Step 1: For given $h_{d}$, choose an upper bound on $h$ satisfying (7), then select this upper bound as the initial value $h_{0}$ of $h$.

Step 2: Set appropriate step lengths, $h_{\text {step }}$ and $\alpha_{\text {step }}$, for $h$ and $\alpha$, respectively. Set $k$ as a counter, and choose $k=1$. Meanwhile, let $h=h_{0}+h_{\text {step }}$ and initial value $\alpha_{0}$ of $\alpha$ equals to $\alpha_{\text {step }}$.

Step 3: Let $\alpha=k \alpha_{\text {step }}$, if inequality (7) is feasible, go to Step 4; otherwise, go to Step 5.

Step 4: Let $h_{0}=h, \alpha_{0}=\alpha, k=1$ and $h=h_{0}+h_{\text {step }}$, go to Step 3.

Step 5: Let $k=k+1$. If $k \alpha_{\text {step }}<1$, then go to Step 3; otherwise, stop.

Remark 4. For Algorithm 1, final $h_{0}$ is the desired maximum of the upper bound of delay $h(t)$ satisfying (7) and $\alpha_{0}$ is the corresponding value of $\alpha$.

Remark 5. Similar to Algorithm 1, we can also find an appropriate scalar $\alpha$, such that the upper bound of delay $0 \leq h(t) \leq \alpha h$, subjecting to (26) attains the maximum.

Remark 6. Similar to Algorithm 1, an algorithm for seeking an appropriate $\alpha$ such that the upper bound of delay $\alpha h \leq h(t) \leq h$, subjecting to (20) and (27) are maximal can be easily obtained.

To show usefulness of our result, let us consider the following numerical examples.

\section{Illustrative Examples}

In this section, five examples are provided to illustrate the advantages of the proposed stability results.

Example 1. Consider the following time delay descriptor systems:

$$
E \dot{x}(t)=A x(t)+B x(t-h(t))
$$

where

$$
E=\left[\begin{array}{ll}
1 & 0 \\
0 & 0
\end{array}\right], A=\left[\begin{array}{cc}
0.6353 & 0.5512 \\
-0.6014 & -1.0998
\end{array}\right], B=\left[\begin{array}{cc}
-0.5 c & 0 \\
0 & -0.1 c
\end{array}\right] .
$$

where $c$ is a scalar. Now, our problem is to estimate the MAUB $\bar{h}$ to keep the stability of system (29).

Solution. Choosing $\alpha=0.5, h_{d}=0.9, c=1, U=\left[\begin{array}{ll}1 & 0\end{array}\right]^{T}$ and applying the LMI Toolbox in MATLAB (with accuracy 0.01 ), this above time delay descriptor system (29) is asymptotically stable for delay time satisfying $h \leq 5.3510$. Table 1 gives comparison results of the criteria in $[6,8,24$, 27] and Theorem 3 for different values of $c$, from which we can also see that the stability criterion in Theorem 3 is less conservative compared to some existing ones $[6,8,24$, 27].

Table 1. Comparison of MAUB $\bar{h}$ using different methods in Example ${ }_{1}\left(h_{d}=0, \alpha=0.5\right)$

\begin{tabular}{cccccc}
\hline$c$ & 1.0 & 1.2 & 1.4 & 1.6 & 2.0 \\
\hline$[6]$ & 2.0362 & 1.7691 & 1.5619 & 1.3977 & 1.1548 \\
{$[24]$} & 2.2750 & 1.9635 & 1.7282 & 1.5438 & 1.2729 \\
{$[27]$} & 2.3290 & 2.0320 & 1.8040 & 1.6220 & 1.3500 \\
{$[8]$} & 2.6892 & 2.4152 & 2.1855 & 1.9947 & 1.7025 \\
Theorem 3 & 8.0559 & 6.2484 & 4.7245 & 3.2869 & 3.0559 \\
\hline
\end{tabular}

Example 2. Consider the following time delay descriptor systems:

$$
E \dot{x}(t)=A x(t)+B x(t-h(t)),
$$

where $E=\left[\begin{array}{ll}1 & 0 \\ 0 & 0\end{array}\right], A=\left[\begin{array}{cc}0.5 & 0 \\ 0 & -1\end{array}\right], B=\left[\begin{array}{cc}-1.1 & 1 \\ -0.1 & -0.7\end{array}\right]$. Now, our problem is to estimate the MAUB $\bar{h}$ to keep the stability of system (30).

Solution. Choosing $U=\left[\begin{array}{ll}0 & 1\end{array}\right]^{T}$ and taking the parameters $h_{d}=0.1$ and $\alpha=0.5$, we get the Theorem 1 remains feasible for any delay time $h \leq 1.9511$. The results for stability conditions in different methods are compared in Table 2. It can be seen that the delay-dependent stability condition in this paper is less conservative than earlier reported ones in the literature [10,21]. Compared with Wang et al. [21] that used 21 LMI variables, we need 11 variables in Theorem 1 the same as [10]. It can be shown that the delay-dependent stability condition in this paper is the best performance.

Table 2. Comparisons of MAUB $\bar{h}$ in Example 2 for different methods $(\alpha=0.5)$

\begin{tabular}{cccc}
\hline Methods & $h\left(h_{d}=0.1\right)$ & $h\left(h_{d}=0.3\right)$ & $\begin{array}{c}\text { Number of } \\
\text { variables }\end{array}$ \\
\hline$[21]$ & 0.9745 & 0.9378 & 21
\end{tabular}




\begin{tabular}{cccc}
{$[10]$} & 0.9745 & 0.9378 & 11 \\
Theorem 1 & 1.9511 & 1.9016 & 11 \\
\hline
\end{tabular}

Example 3. Consider the following time delay descriptor systems:

$$
E \dot{x}(t)=A x(t)+B x(t-h(t))
$$

where $E=\left[\begin{array}{ll}1 & 0 \\ 0 & 0\end{array}\right], A=\left[\begin{array}{cc}0.5 & 0 \\ -1 & -1\end{array}\right], B=\left[\begin{array}{cc}-1 & 0 \\ 0 & 0\end{array}\right]$.

Now, our problem is to estimate the MAUB $\bar{h}$ to keep the stability of system (31).

Solution. Choosing $U=\left[\begin{array}{ll}1 & 0\end{array}\right]^{T}, h_{d}=0$, and applying the LMI Toolbox in MATLAB (with accuracy 0.01), this above time delay descriptor system (31) is asymptotically stable for delay time satisfying $h \leq 2.4052$. Table 3 lists the results compared with $[7,11,14,23-27,30]$. It can be seen from Table 3 that the maximum admissible upper bound (MAUB) $\bar{h}$ by using Theorem 3 is the largest with the fewest variables computed. As Fig. 1 shows, the simulation of the above system (31) for $h=2.4$ with the initial state $\left[\begin{array}{ll}-1 & 1\end{array}\right]^{T}$.

Table 3. Comparison of MAUB $\bar{h}$ in Example 3 for different methods
$(\alpha=0.5)$

\begin{tabular}{ccc}
\hline Methods & MAUB $(h)$ & Number of variables \\
\hline$[23]$ & 1.1547 & 53 \\
{$[25]$} & 1.1547 & 33 \\
{$[7]$} & 1.1547 & 24 \\
{$[24]$} & 1.1547 & 17 \\
{$[26]$} & 1.2011 & 13 \\
{$[11]$} & 1.1898 & 13 \\
{$[30]$} & 1.2052 & 33 \\
{$[27]$} & 1.2060 & 21 \\
{$[14]$} & 2.3092 & 11 \\
Theorem 3 & 2.4052 & 11 \\
\hline
\end{tabular}

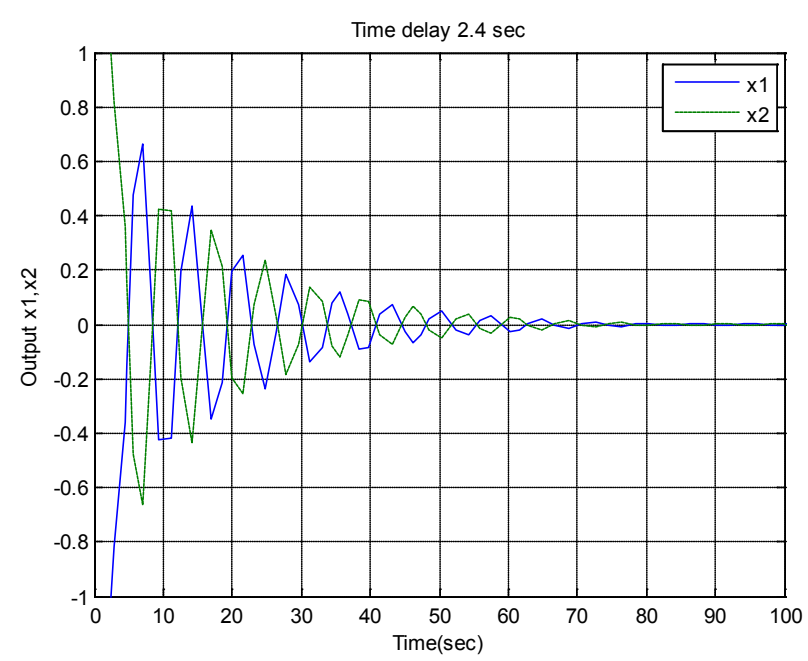

Example 4. For convenience of comparison, consider the following time-delay descriptor system $[3,6,18,20]$.

$$
E \dot{x}(t)=A x(t)+B x(t-h(t))
$$

where $E=\left[\begin{array}{cc}-1 & 1 \\ 1 & -1\end{array}\right], A=\left[\begin{array}{cc}-1 & 0 \\ 0 & -1\end{array}\right], B=\left[\begin{array}{cc}1 & -1 \\ -1 & 1\end{array}\right]$.

Now, our problem is to estimate the MAUB $\bar{h}$ to keep the stability of descriptor system (32).

Solution. In this example, we choose $U=\left[\begin{array}{ll}0 & 1\end{array}\right]^{T}$. For $h_{d}=0$ the system (32), we are able to find a feasible solution for the set of LMIs for any $h \leq 1.7859$. This means that the MAUB $(\bar{h})$ under which the system is uniformly asymptotically stable is 1.7859 . Table 4 lists the MAUB $\bar{h}$ as judged by the criteria in $[3,6,18,20]$. It can be seen that the delay-dependent stability condition in this paper is less conservative than earlier reported ones in the literature $[3,6,18,20]$. We also know that with fewer matrix variables the stability results obtained in Theorem 3 is less conservative than the one in $[3,6,18,20]$. Using these data, a simulation program has been written in Matlab. As Fig. 2 shows, the simulation of the above system (32) for $h=1.78$ with the initial state $\left[\begin{array}{ll}-1 & 1\end{array}\right]^{T}$.

Table 4. MAUB $(\bar{h})$ in example 4 for different methods $(\alpha=0.5)$

\begin{tabular}{ccc}
\hline Methods & MAUB $(h)$ & Number of variables \\
\hline$[6]$ & 1.150 & 9 \\
{$[3]$} & 1.1547 & 7 \\
{$[18,20]$} & 1.1547 & 5 \\
Theorem 3 & 1.7859 & 5 \\
\hline
\end{tabular}

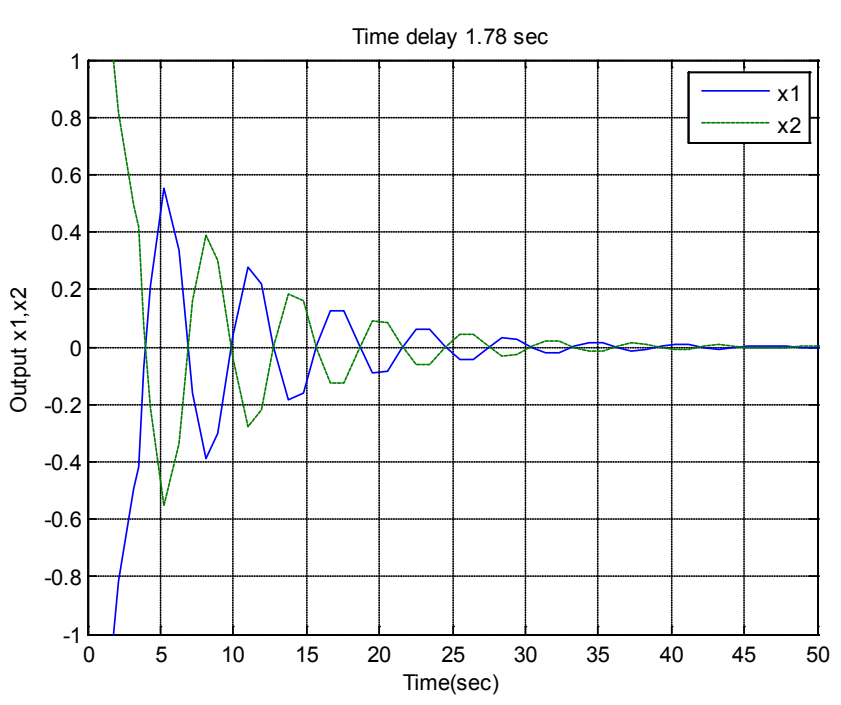

Figure 2. The simulation of the example 4 for $h=1.78 \mathrm{sec}$

Example 5. Consider the following descriptor system:

Figure 1. The simulation of the example 3 for $h=2.4 \mathrm{sec}$ 


$$
\left[\begin{array}{ll}
1 & 0 \\
0 & 1
\end{array}\right] \dot{x}(t)=\left[\begin{array}{cc}
-2 & 0 \\
0 & -0.9
\end{array}\right] x(t)+\left[\begin{array}{cc}
-1 & 0 \\
-1 & -1
\end{array}\right] x(t-h(t))
$$

where $0 \leq h(t) \leq h$.

Solution. When $h$ is a constant. For different methods, the computed MAUB $\bar{h}$, which guarantee the stability of system (33) are listed in Table 5. It is clear that the result obtained by Theorems 3 and 4 are less conservative than the ones by the methods in [31], and the result obtained by Theorems 3 and 4 outperforms the existing ones [31].

Table 5. Comparisons of $\operatorname{MAUB}(\bar{h})$ in example 5 for different
methods.

\begin{tabular}{cc}
\hline Method & $\operatorname{MAUB}(h)$ \\
\hline$[31]$ ( Theorem 1) $(\alpha=0.63)$ & 1.602 \\
[31] (Theorem 2) $(\alpha=0.7)$ & 2.065 \\
Theorem 3 $(\alpha=0.6)$ & 2.1679 \\
Theorem 4 $(\alpha=0.6)$ & 2.1565 \\
\hline
\end{tabular}

\section{Conclusion}

In this paper, a delayed decomposition approach has been developed to investigate the stability of descriptor systems with a time-varying delay. By developing a delayed decomposition approach, the information of the delayed plant states can be taken into full consideration, and new delay-dependent sufficient stability criteria are obtained in terms of linear matrix inequalities (LMls). The stability condition is expressed in terms of easily computable LMIs. It is proved that the obtained results are less conservative than some existing ones. Meanwhile, the computational complexity of the new stability criteria is reduced greatly since fewer decision variables are involved. An algorithm of seeking appropriate tuning parameter is also presented. Numerical examples have illustrated the effectiveness of the proposed methods.

\section{REFERENCES}

[1] S. Boyd, L. E. Ghaoui, E. Feron, V. Balakrishnan, Linear Matrix Inequalities in Systems and Control Theory, SIAM, Philadelphia, PA(1994).

[2] L. Dai, Singular Control Systems. Springer-Verlag, New York (1989).

[3] Y. Ding, S. W. Mei, X. Wang, Novel stability criteria of nonlinear uncertain systems with time-varying delay, Abstract and Applied Analysis, pp.1-16, doi:10.1155/ 2011/969674(2011)

[4] Y. Feng, X. Zhu, Q. Zhang, Delay dependent stability criteria for singular time delay systems, Acta Automatica
Sinica, Vol. 36, pp. 433-437, 2010.

[5] E. Fridman, Stability of linear descriptor systems with delay: a Lyapunov-based approach, Journal of Mathematical Analysis and Applications, Vol. 273, pp.24-44, 2002.

[6] E. Fridman, U. Shaked, $H_{\infty}$ control of linear state-delay descriptor systems: an LMI approach, Linear Algebra and its Applications, Vol. 351, pp.271-302, 2002.

[7] H. L. Gao, B. Xu, Delay-dependent state feedback robust stabilization for uncertain singular time delay systems, Journal of Systems Engineering and Electronics, Vol.19, pp.758-765, 2008.

[8] Z. H. Jiang, W. H. Gui, Y. F. Xie, A less conservative delay-dependent stability for uncertain singular linear systems with time-delays, Proceeding of the 8th World Congress on Intelligent Control and Automation, pp. 3673-3678, 2010.

[9] F. L. Lewis, A survey of linear singular systems. Circuits Systems Signal Processing, Vol. 5, pp.3-36, 1985.

[10] F. Li, X. Zhang, A delay-dependent bounded real lemma for singular LPV systems with time-variant delay, International Journal of Robust and Nonlinear Control, Vol. 22, pp.559-574, 2012.

[11] S. J. Liang, J. Cheng, M. Huang, A delay decomposition approach to stability analysis of singular systems with time-varying delay, Proceeding of the 2009 International Conference on Test and Measurement, pp.110-114, 2009.

[12] L. L. Liu, J. G. Peng, B. W. Wu, On parameterized Lyapunov-Krasovskii functional techniques for investigating singular time-delay systems, Applied Mathematics Letters, Vol. 24, pp.703-708, 2010.

[13] P. L. Liu, Further results on the exponential stability criteria for time delay singular systems with delay-dependence, International Journal of Innovative Computing Information and Control, Vol. 8, pp.4015-4024, 2012.

[14] P. L. Liu, Further results on the stability analysis of singular systems with time-varying delay: A delay decomposition approach, International Journal of Analysis ID 721407, pp.1-11, 2013.

[15] P. L. Liu, A delay decomposition approach to stability analysis of uncertain systems with time-varying delays, ISA Transactions, Vol. 51, pp.694-701, 2012.

[16] P. L. Liu, A delay decomposition approach to stability analysis of neutral systems with time-varying delay, Applied Mathematical Modeling, Vol. 37, pp. 5013-5026, 2013.

[17] P. L. Liu, State feedback stabilization of time-varying delay uncertain systems: A delay decomposition approach, Linear Algebra and its Applications, Vol. 438, pp.2188 -2209, 2013.

[18] P. L. Liu, Delay-range-dependent robust stability for uncertain singular systems with interval time-varying delays, Universal Journal of Control and Automation, Vol. 1, No.1, pp. 1-9, 2013.

[19] H. Su, X. Ji, J. Chu, Delay-dependent robust control for uncertain singular time-delay systems, Asian Journal of Control, Vol. 8, pp. 180-189, 2006.

[20] C. H. Wang, New delay-dependent stability criteria for 
descriptor systems with interval time delay, Asian Journal of Control, Vol. 14, pp.197-206, 2012.

[21] H. J. Wang, A. K. Xue, R. Q. Lu, J. H. Wang, Delay-dependent robust stability and stabilization for uncertain singular system with time-varying delay, Proceedings of the 2008 American Control Conference, Seattle, WA, 11-13 June, pp. 3626-3631, 2008.

[22] X. Wu, Y. Wang, L. Huang, Y. Zuo, Robust stability analysis of delayed Takagi-Sugeno fuzzy Hopfield neural networks with discontinuous activation functions, Cognitive Neurodynamics, Vol. 4, pp. 347-354, 2010.

[23] Z. Wu, W. Zhou, Delay-dependent robust $H_{\infty}$ control for uncertain singular time-delay systems, IET Control Theory and Applications, Vol. 1, pp.1234-1241, 2007.

[24] S. Xu, J. Lam, Y. Zou,An improved characterization of bounded realness for singular delay systems and its applications, International Journal of Robust and Nonlinear Control, Vol. 18, pp.263-277, 2008.

[25] D. Yue, Q. L. Han, A delay-dependent stability criterion of neutral systems and its application to a partial element equivalent circuit model, IEEE Transactions on Circuits and Systems-II: Express Briefs, Vol. 51, pp.685-689, 2004.
[26] S. Zhu, C. Zhang, Z. Cheng, J. Feng, Delay-dependent robust stability criteria for two classes of uncertain singular time-delay systems, IEEE Transactions on Automatic Control, Vol. 52, pp.880-885, 2007.

[27] S. Zhu, Z. Li, C. Zhang, Delay decomposition approach to delay-dependent stability for singular time-delay systems, IET Control Theory and Applications, Vol. 4, pp.2613-2620, 2010.

[28] S. Q. Zhu, Z. L. Chen, J. Feng, Delay-dependent robust stability criterion and robust stabilization for uncertain singular time-delay systems, Proceedings of American Control Conference, Portland, USA, pp.2839-2844, 2005.

[29] X. L. Zhu, G. H. Yang, Jensen integral inequality approach to stability analysis of continuous-time systems with time varying delay, IET Control Theory and Applications, Vol. 2, pp.524-534, 2008.

[30] X. L. Zhu, G. H. Yang, New results of stability analysis for singular time-delay systems, Proceedings of the 2009 American Control Conference, Hyatt Regency Riverfront, St. Louis, MO, USA, FrB09.4, pp.4905-4908, 2009

[31] X. L. Zhu, G. H. Yang, New results of stability analysis for systems with time-varying delay, International Journal of Robust and Nonlinear Control, Vol. 20, pp.596-606, 2010. 